\title{
Medical Behavior in Cardiorespiratory Arrest before and After Simulation Based on Advanced Cardiac Life Support (ACLS) Course
}

\author{
Douglas Marostica Catolino ${ }^{(1)}$ and José Knofholz \\ Pontíficia Universidade Católica do Paraná, Curitiba, PR - Brazil
}

\section{Abstract}

Background: The Advanced Cardiac Life Support (ACLS) course is designed to teach cardiovascular emergency, aiming to promote a harmonious and synchronized work of the entire hospital team, making the multidisciplinary job more effective in the execution of cardiopulmonary resuscitation (CPR).

Objective: To compare the effectiveness of CPR performed between physicians trained on ACLS and non-trained physicians.

Methods: A questionnaire was applied to physicians working at the emergency room of hospitals in Curitiba, state of Paraná, whose resolution required theoretical and practical knowledge about CPR. For analysis, descriptive statistics and Fisher's association analysis were used, and the medians of the groups were evaluated by MannWhitney/Kruskal-Wallis with significance of $5 \%$.

Results: Thirty-four physicians were volunteers, of whom 20 had taken the ACLS course (Group A) and 14 had not (Group B). The trained physicians obtained the highest median (4.00 vs. 3.00, p = 0.06) of correct answers. Group A scored at least 3 of the 5 questions in the questionnaire, showing better performance than Group B $(\mathrm{OR}=6.75,95 \% \mathrm{CI}, 1.1<\mathrm{OR}<41.0, \mathrm{p}=0.04)$. The year of the course did not significantly change the performance in the questionnaire.

Conclusion: It is suggested that the ACLS course was effective in qualifying physicians to handle situations of cardiorespiratory arrest properly, which was reproduced by the better performance in the resolution of the questionnaire. It is believed that when the sample of volunteers is increased, the trends found materialize the other hypotheses proposed. (Int J Cardiovasc Sci. 2020; 33(2):151-157)

Keywords: Cardiopulmonary, Resuscitation; Advanced Cardiac Life Support/training; Heart Arrest.

\section{Introduction}

Advanced Cardiac Life Support (ACLS) is a course that has occurred in Brazil since 1996 and is designed to teach cardiovascular emergency. It starts with the simulation of real-life situations through a standard way of teaching an ideal clinical management professional in the medical field. The course structure is periodically reviewed according to new versions provided by the American Heart Association (AHA) guidelines. ${ }^{1}$ In recent years, cardiovascular emergency courses have been applied to a larger number of physicians working in emergency rooms or elsewhere. ${ }^{2}$ It is known that since its introduction, ACLS has undergone a series of conceptual and structural changes. The course has turned its focus on solving cases with defined objectives, and practical activities were emphasized. Modifications included theoretical updates according to evidencebased medicine and guidelines applied to the new teaching approach. ${ }^{1}$ 
Doctors' updating is crucial for adequate management of cardiorespiratory arrest. Training and continued education on cardiopulmonary resuscitation (CPR), ${ }^{3,4}$ since success in cardiac arrest management requires technical and scientific efficiency, as well as adequate team training. ${ }^{5}$ Cardiorespiratory arrest care should be considered mandatory and priority knowledge for all health professionals, regardless of their specialty. It requires adequate infrastructure with frequent updates to basic life support conduct for an effective, harmonious and synchronized work of the multidisciplinary team. ${ }^{6,7}$

This work aims to identify the effectiveness of ACLS course in enabling physicians to handle cardiovascular emergency situations. It was based on the assumption that ACLS essentially seeks to standardize the actions in a cardiorespiratory arrest situation, so the patients may receive the best treatment. ${ }^{1}$

The importance of this study is remarkable because if the alleged increase in effectiveness after ACLS training is confirmed, more hospitals or educational institutions would appreciate the application of the course and invest in the training of their professionals and students. This investment would be reflected in the patients with cardiorespiratory arrest, who would most likely be assisted by successful resuscitation teams.

\section{Methods}

\section{Data collection}

The ACLS efficacy analysis was performed by distributing a questionnaire to physicians working at the emergency room of hospitals in the city of Curitiba, state of Paraná. The questionnaire was to be answered on a volunteering basis and included 5 multiple choice questions. It contained an approach involving theoretical and practical knowledge about cardiopulmonary resuscitation (Supplementary 1). In addition to the questions, additional data were collected such as the physician's year of training, age, institution of graduation, medical specialty, employing hospital and participation in the ACLS course, as well as the year in which such training took place. Volunteering physicians answered the survey in front of a researcher in the emergency room. This was to avoid any potential consultation or communication among the volunteers, which could add bias to the results. In total, 34 physicians participated in the study. There was no specific criterion for determining the sample size. The number of volunteers was exclusively due to the number of physicians willing to participate in the study that met the inclusion criteria during the data collection period.

Once the data were collected, the main focus of the discussion was the comparison between the performance of volunteers who had attended the ACLS course versus the performance of those who did not, seeking to analyze the effectiveness of the course. Also, the retention of knowledge in the short and long term was examined by contrasting the results of the volunteers participating in the course to the year in which such training occurred.

\section{Statistical analysis}

The epidemiological data collected in the questionnaire was evaluated by descriptive statistics, and normality check was performed using the Kolmogorov-Smirnov test. The variables were represented by medians and values of the first and third quartiles. For the association analysis, independence tests were performed between the groups using Fisher's test with odds ratio calculation, and 95\% confidence interval using the SISA program package. The analysis between the groups and the median number of correct questions were performed using the MannWhitney/Kruskal-Wallis tests with odds ratio calculation, in the GraphPad Prism program. P values lower than 0.05 were considered significant.

\section{Results}

The questionnaire was distributed to 34 volunteer physicians working at the emergency room of hospitals in Curitiba. Of this total, 20 had attended an ACLS course, while 14 had not. In order to facilitate exposure, the group of physicians who attended the ACLS

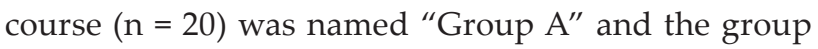
of physicians who had never attended an ACLS course $(n=14)$ was called "Group B". Since the data followed a non-normal distribution pattern, nonparametric statistical tests were performed.

Regarding the number of correct answers in the questionnaire, it could be observed that the groups (physicians who have attended an ACLS course versus physicians who have never attended such course) tend to be different, with $\mathrm{p}$ value of 0.06 (Figure 1). Group A obtained a median score greater than Group B (4.00 vs. 3.00). However, this difference was not statistically validated $(p=0.06)$. In addition, it was observed that Group A scored at least 3 of the 5 questions in the questionnaire, while Group B showed inferior 
performance $(\mathrm{OR}=6.75,95 \% \mathrm{CI}, 1.1<\mathrm{OR}<41.0$, $\mathrm{p}=0.04)$ (Table 1). Of the 20 physicians who were part of Group A, only 2 scored less than 3 questions, so $90.00 \%$ of the sample answered correctly the majority of the questionnaire. In comparison, of the 14 professionals from Group B, 6 (42.86\%) scored less than half of the test, while only $57.14 \%$ made 3 or more questions.

To investigate whether the time since the ACLS course to the date of the questionnaire could affect the performance of the volunteers, Group A was subdivided into two parts, according to the year of the course: (i) volunteers who took the ACLS course in the year of 2016; (ii) volunteers who took the ACLS course prior to the year of 2016 (Figure 2). None of the volunteers took the course during the year of 2017. No significant difference was noticed between the groups $(\mathrm{p}=0.52)$. In each group, only 1 individual who took the course scored less than 3 questions. Thus, it could be observed that even physicians who were trained with outdated guidelines were able to achieve similar performance to that of recently trained professionals, and better than group $B(p=0.04)$.

\section{Discussion}

A questionnaire was distributed to physicians on duty at emergency units of hospitals in Curitiba, where 34 professionals were volunteers. Of these, 20 physicians had taken an ACLS course (group A), and 14 had not (group B).

Regarding the number of correct answers in the questionnaire, it was observed that group A obtained median score greater than Group B. However, this difference was not significant $(p=0.06)$. It is believed that this tendency would be confirmed by an increase in the sample number, which was not possible in this study, since it was difficult to recruit volunteers who fulfilled the inclusion criteria (physicians on duty in emergency departments of hospitals in the city of Curitiba during the data collection period). The researchers chose to look for volunteer physicians working at emergency care units to participate in the study because they believed that in that environment, cardiac arrest situations were found more frequently than in other medical settings, such as outpatient clinics, for example. Therefore, it would be indispensable for the professional to be knowledgeable about the management of CPR for quality emergency care. However, one limitation to sample size was the typical agitation of emergency room units, as well as physicians' allegations of insufficient time to answer the questionnaire questions with the utmost attention. Still, to avoid consultation or communication between professionals, they were required to answer the questionnaire in the presence of a researcher in charge, which also reduced the sample.

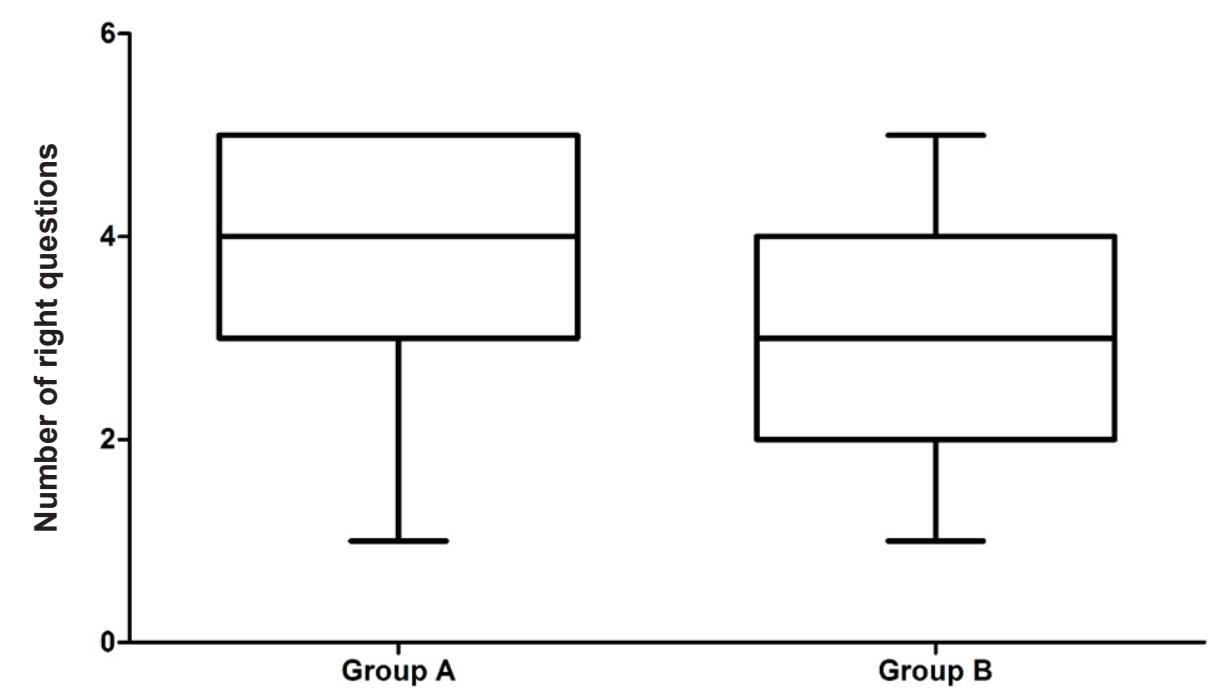

Figure 1 - Performance of the groups evaluated for the median number of correct answers in the questionnaire. Group A corresponds to the doctors who had taken an ACLS course; Group B corresponds to those who had not $(p=0.06)$. 


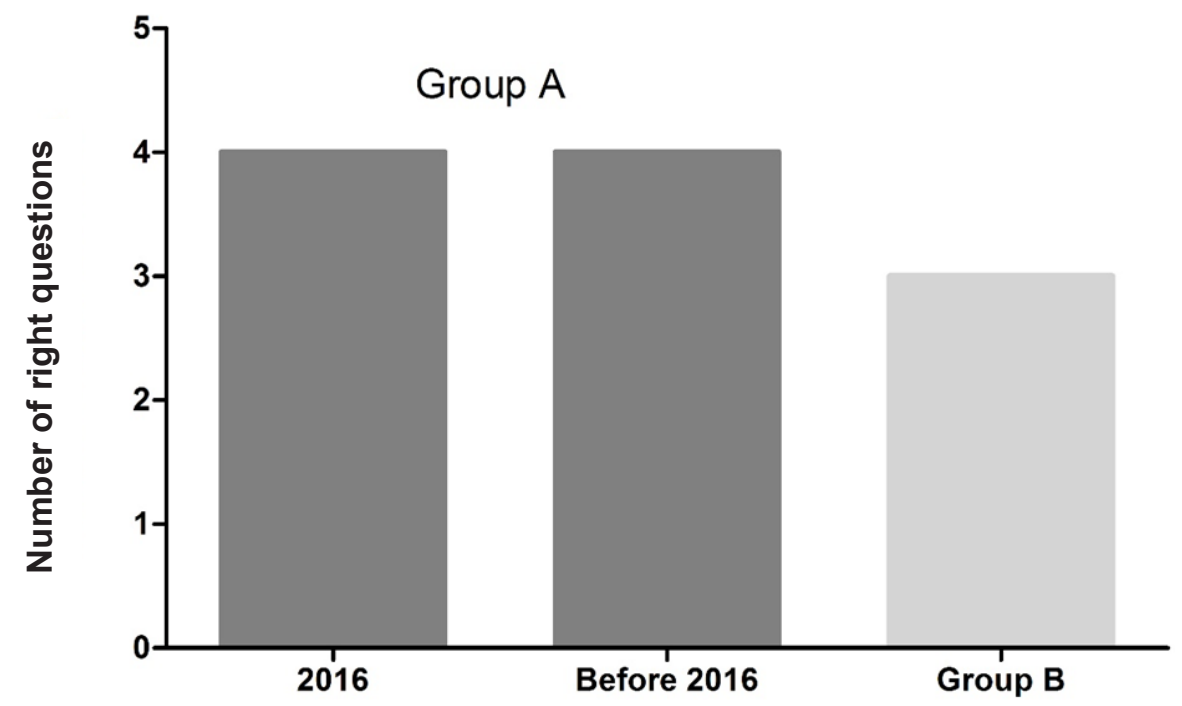

Figure 2 - Median number of correct answers in the questionnaire of groups A and B. Group A was subdivided into two according to the year of the course: (i) volunteers who took ACLS training in 2016; (ii) volunteers who took ACLS training before 2016. No significant difference was found in the number of correct answers in the subgroups, both of which reached a median higher than group B.

It was observed that group A scored at least 3 of the 5 questions of the questionnaire, whereas group B showed inferior performance, which suggests that physicians who took the course have a chance to show better performance in the questionnaire than those who never did.

The aim of this study was also to investigate whether the time since the ACLS course to the date of the questionnaire could affect the performance of volunteers. For this purpose, Group A was subdivided into two parts: volunteers who took ACLS training in 2016 and volunteers who had taken training previously. It was decided to organize the volunteers in this manner because the last modification of the guidelines was published in 2015 by the American Heart Association (AHA). No significant difference was observed between the groups, so the ACLS course allowed a large number of correct answers regardless of the year of training ( $p=$ 0.52 ). Even physicians who were trained with outdated guidelines were able to achieve performance similar to that of newly trained practitioners - still better than the group that never trained $(p=0.04)$. It is suggested that physicians trained under old protocols were able to update CPR concepts. Besides that, regardless of the year of training, knowledge retention was reflected by the better performance in the questionnaire.
It is assumed that the effectiveness of the course was able to promote significant changes in the approach of professionals trained on CPR, resulting in better medical care and longer survival of patients under CRP. It is hoped that this analysis may foster the importance of training and prior experience with real situations regarding the effectiveness in resuscitation of patients in cardiorespiratory arrest.

Thus, it is possible to recognize the effectiveness of the ACLS course in promoting better performance of the

Table 1 - Number of correct answers of different groups evaluated

\begin{tabular}{lccc}
\hline Scores & $\begin{array}{c}\text { Group A } \\
\mathbf{n}=\mathbf{2 0}\end{array}$ & $\begin{array}{c}\text { Group B } \\
\mathbf{n}=\mathbf{1 4}\end{array}$ & p value ** \\
\hline $25 \%$ quartile & 3.00 & 2.00 & NA \\
Median & 4.00 & 3.00 & 0.06 \\
$75 \%$ quartile & 5.00 & 4.00 & NA \\
$\geq 3 / 5^{*}$ & 18.00 & 8.00 & 0.04 \\
\hline
\end{tabular}

${ }^{*}$ ) At least three scores on five questions answered in the questionnaire; (**) The difference between medians was evaluated by Mann-Whitney test, and the difference between the number of correct answers using Fisher's test. In bold, statistically significant $(<0.05)$. NA: not applicable. 
professionals who answered the questionnaire, which reflects greater acquisition of knowledge and skills developed with the training.

\section{Discussion}

Considering the results obtained, it is proposed that the ACLS course was effective in qualifying physicians to deal more adequately with cardiorespiratory arrest situations - which was reproduced by better performance in the questionnaire - fulfilling the objective of this study. The year of course completion did not significantly change the volunteers' performance in solving the questions, pointing out adequate retention of knowledge and probable professional update of those physicians trained on non-recent protocols.

Although the reduced sample of professionals who volunteered to participate in the study had limited a better statistical result of some variables, it is believed that once the sample is increased, the trends found would support the hypotheses proposed.

\section{Acknowledgments}

To Pontifícia Universidade Católica do Paraná for this opportunity. To Tamyres Mingorance Carvalho for assistance in statistics.

\section{References}

1. Garrido FD, Romano MMD, Schmidt A, Pazin-Filho A. Can course formatinfluence the performance of students in an advanced cardiac life support(ACLS) program? Braz J Med Biol Res. 2011;44(1):23-8.

2. Lima E Jr, Knopfholz J, Menini CM. Stress during ACLS courses: is it important for learning skills? Arq Bras Cardiol. 2002;79(6):589-92.

3. Knopfholz J, Lima Jr E, Ribeiro APF, Boros GAB, Claviço J, Teider LD, et al. Management of cardiologic emergencies in general hospitals in the state of Paraná. Rev Bras Clin Med. 2012;10(6):499-502.

4. Duarte RN, Fonseca AJ Da. Diagnóstico e tratamento de parada cardiorrespiratória : avaliação do conhecimento teórico de médicos em hospital geral. Rev Bras Ter Intensiva. 2010;22(4):153-8.

\section{Author contributions}

Conception and design of the research: Catolino DM, Knofholz J. Acquisition of data: Catolino DM. Analysis and interpretation of the data: Catolino DM. Statistical analysis: Catolino DM. Writing of the manuscript: Catolino DM. Critical revision of the manuscript for intellectual content: Knofholz J.

\section{Potential Conflict of Interest}

No potential conflict of interest relevant to this article was reported.

\section{Sources of Funding}

There were no external funding sources for this study.

\section{Study Association}

This study is not associated with any thesis or dissertation work.

\section{Ethics approval and consent to participate}

This study was approved by the Ethics Committee of the PUC-PR under the protocol number 1709.529. All the procedures in this study were in accordance with the 1975 Helsinki Declaration, updated in 2013. Informed consent was obtained from all participants included in the study.

5. Gomes AG, Garcia AM, Schmidt A, Mansur AP, Vianna CB, Ferreira D, et al. Diretriz de Apoio ao Suporte Avançado de Vida em CardiologiaCódigo Azul-Registro de Ressuscitação Normatização do Carro de Emergência. Arq Bras Cardiol. 2003;81(SuplIV):1-14.

6. Feitosa-Filho GS, Feitosa GF, Guimarães HP, Lopes RD, Moraes Júnior R, Souto FA, et al. Update on cardiopulmonary resuscitation: what changed with the new guidelines.. Rev Bras Ter Intensiva. 2006;18(2):177-85.

7. Timerman A, Sauaia N, Piegas LS, Ramos RF, Gun C, Santos ES, et al Fatores Prognósticos dos Resultados de Ressuscitação Cardiopulmonar em um Hospital de Cardiologia. Arq Bras Cardiol. 2001;77(2):142-51. 
Supplementary File. Questionnaire about cardiopulmonary resuscitation.

Age: University: Year of graduation:

Medical Specialty:

Employing Hospital:

Have you ever attended an ACLS course? Yes _ No_ If yes, year of participation:

1. In a cardiorespiratory arrest in an adult patient not in a hospital environment, according to the current guidelines, what is the correct sequence that must be followed by a health professional after local safety?

A. Check responsiveness, contact the emergency service/get an Automated External Defibrillator (AED), check breathing and pulse sequentially, ventilate, start chest compressions.

B. Check responsiveness, start chest compressions, contact the emergency service/get an Automated External Defibrillator (AED), check breathing and pulse simultaneously.

C. Check responsiveness, contact the emergency service/get an Automated External Defibrillator (AED), check breathing and pulse simultaneously, start chest compressions.

D. Contact the emergency service/get an Automated External Defibrillator (AED), check responsiveness, check breathing and pulse sequentially, start chest compressions.

E. Contact the emergency service/get an Automated External Defibrillator (AED), check responsiveness, start chest compressions, check breathing and pulse simultaneously.

\section{About the Cardiopulmonary Resuscitation (CPR) technique, mark the INCORRECT statement:}

A. In 2015, the frequency of compressions was modified to the range of 100 to $120 / \mathrm{min}$.

B. In 2015, the depth of adult compression was modified to at least 2 inches $(5 \mathrm{~cm})$, not to exceed 2.4 inches $(6 \mathrm{~cm})$.

C. Adequate ventilation would be 2 ventilations for every 30 compressions regardless of the number of professionals involved in CRP care, with each breath administered in one second in order to induce chest elevation.

D. After advanced airway management, ventilation should be performed at a frequency of 1 time every 3 seconds.

E. Cricoid compression should not be routinely used in orotracheal intubation during CPR.

\section{About the Cardiopulmonary Resuscitation (CPR) technique, mark the INCORRECT statement:}

A. Hyperkaliemia, acidosis and tricyclic intoxication are treated with sodium bicarbonate in the course of a CPR.

B. The defibrillator should be used as soon as it is available.

C. When the defibrillator arrives, if the pace is shocking, after 1 shock, do CPR immediately for about 5 minutes.

D. Vasopressin in CPR can be used in combination with epinephrine or alone.

E. Epinephrine should be given as soon as possible after initiation of CPR due to an initial non-shockable rhythm.

\section{Determine the following assertions as true $(\mathrm{T})$ or false $(\mathrm{F})$ and choose the right answer:}

i. Synchronized shock (360 J) every $2 \mathrm{~min}$ is the best course for ventricular fibrillation/pulseless ventricular tachycardia.

ii. The recommended dose of epinephrine is $1 \mathrm{mg}$ every 3 to 5 minutes, while amiodarone is $300 \mathrm{mg}$ in the first dose and $150 \mathrm{mg}$ in the second dose.

iii. Immediately after the shock, if the patient returns to sinus rhythm, it is not necessary to apply compressions, being able to leave for post-CPR care

iv. Ventricular fibrillation is the main cause of $\mathrm{CPR}$, while asystole represents the worse prognosis. 
v. If in capnography $\mathrm{PETCO}_{2}$ is $<10 \mathrm{mmHg}$, it means that CPR is good quality.
a. F, F, F, T, T.
b. F, T, F, F, F.
c. T, T, F, F, F.
d. F, T, F, T, F.
e. T, F, F, T, T.

5. Male, 59 years old, diabetic, smoker, had cardiopulmonary resuscitation stopped in a non-hospital setting. After compression starts and the Automated External Defibrillator (AED) arrives, there is no pulse, and the following ECG rhythm occurs:

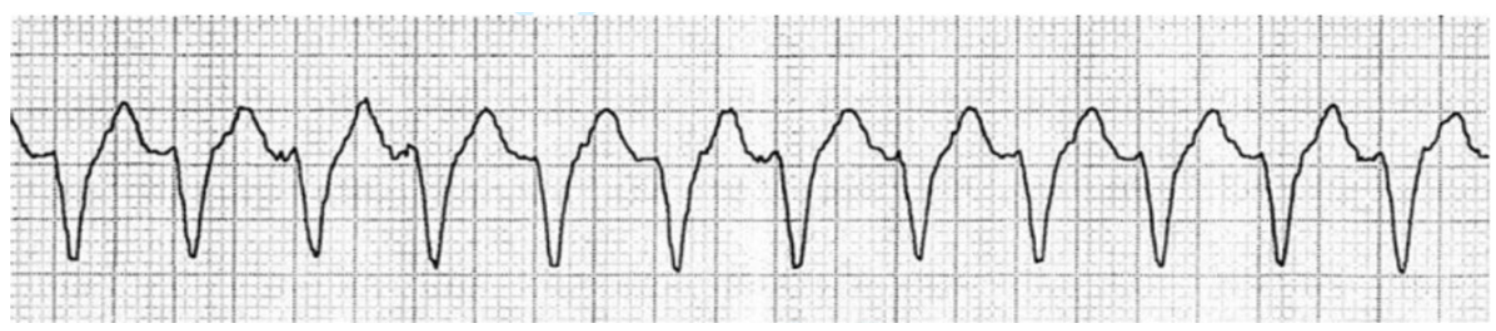

The conduct for this case, according to current guidelines of the American Heart Association regarding ACLS (Advanced Cardiac Life Support), would be:

A. Apply a shock and restart CPR immediately for about 2 minutes. If the rhythm changes to non-shockable, administer amiodarone $300 \mathrm{mg}$ IV/10.

B. Apply a shock and restart CPR immediately for about 2 minutes. If the rhythm remains, apply a second shock, then epinephrine $1 \mathrm{mg} I \mathrm{~V} / 10$.

C. Apply a shock and restart CPR immediately for about 2 minutes. If the rhythm remains, apply a second shock, then epinephrine $1 \mathrm{mg}$ and amiodarone $300 \mathrm{mg}$ IV/10.

D. Restart CPR immediately for about 2 minutes, then apply epinephrine $1 \mathrm{mg}$ IV/10.

E. Restart CPR immediately for about 2 minutes, then apply epinephrine $1 \mathrm{mg}$ and amiodarone $300 \mathrm{mg}$ IV/10. 